

\title{
A subspace theorem for subvarieties
}

\author{
Min Ru and Julie Tzu-Yueh Wang
}

\begin{abstract}
We establish a height inequality, in terms of an (ample) line bundle, for a sum of subschemes located in $\ell$-subgeneral position in an algebraic variety, which extends a result of McKinnon and Roth (2015). The inequality obtained in this paper connects the result of McKinnon and Roth (the case when the subschemes are points) and the results of Corvaja and Zannier (2004), Evertse and Ferretti (2008), Ru (2017), and Ru and Vojta (2016) (the case when the subschemes are divisors). Furthermore, our approach gives an alternative short and simpler proof of McKinnon and Roth's result.
\end{abstract}

\section{Introduction and statements}

McKinnon and M. Roth [2015] introduced the approximation constant $\alpha_{x}(L)$ to an algebraic point $x$ on an algebraic variety $V$ with an ample line bundle $L$. The invariant $\alpha_{x}(L)$ measures how well $x$ can be approximated by rational points on $V$ with respect to the height function associated to $L$. They showed that $\alpha_{x}(L)$ is closely related to the Seshadri constant $\epsilon_{x}(L)$ measuring the local positivity of $L$ at $x$. They also showed that the invariant $\alpha_{x}(L)$ can be computed through another invariant $\beta_{x}(L)$ in the height inequality they established (see Theorem 5.1 and Theorem 6.1 in [McKinnon and Roth 2015]). By computing the Seshadri constant $\epsilon_{x}(L)$ for the case of $V=\mathbb{P}^{1}$, their result recovers Roth's theorem, so the height inequality they established can be viewed as a generalization of this theorem to arbitrary projective varieties.

In this paper, we provide a simpler proof of the above results. Furthermore, we extend the results from the points of a projective variety to subschemes. The generalized result in terms of subschemes connects, as well as gives a clearer explanation to, the above mentioned result of McKinnon and Roth with the recent Diophantine approximation results in terms of the divisors obtained in [Corvaja and Zannier 2004; Evertse and Ferretti 2008; Levin 2014; Ru and Vojta 2016; Ru 2017].

We now state our result. Let $V$ be a projective variety defined over a number field $k$.

MSC2010: primary 11J97; secondary 11J87, 14G05.

Keywords: Schmidt's subspace theorem, Roth's theorem, Diophantine approximation, Vojta's conjecture. 
Definition 1.1. Let $L$ be a line bundle over $V$ with $h^{0}(V, N L) \geq 1$ for $N$ big enough. Let $Y$ be a proper closed subscheme of $V$ and $\pi: \widetilde{V} \rightarrow V$ be the blow-up along $Y$, and $E$ be the exceptional divisor. We define

$$
\beta_{L, Y}:=\liminf _{N \rightarrow \infty} \frac{\sum_{m=1}^{\infty} h^{0}\left(\tilde{V}, N \pi^{*} L-m E\right)}{N \cdot h^{0}(V, N L)} .
$$

Remark 1.2. (a) If $Y$ is an effective Cartier divisor, then the blow-up is an isomorphism. Without loss of generality, we let $\pi$ be the identity map, $\widetilde{V}=V$ and $E=Y$.

(b) Let $D$ be an effective divisor on $V$, we define $\beta_{D, Y}:=\beta_{\mathcal{O}(D), Y}$, where $\mathcal{O}(D)$ is the line sheaf associated to $D$.

(c) In the case when $L$ is big, the $\lim _{N \rightarrow \infty}$ in the definition above exists. Indeed (see [McKinnon and Roth 2015, pp. 544-545]), we have

$$
\beta_{L, Y}=\int_{0}^{\gamma_{\text {eff }}} \frac{\operatorname{Vol}\left(L_{\gamma}\right)}{\operatorname{Vol}(L)} d \gamma
$$

where $L_{\gamma}:=\pi^{*} L-\gamma E$ and $\gamma_{\text {eff }}=\sup \left\{\gamma \geq 0 \mid L_{\gamma}\right.$ is effective $\}$.

Definition 1.3. We say that the closed subschemes $Y_{1}, \ldots, Y_{q}$ of a projective variety $V$ are in $\ell$-subgeneral position if, for any $x \in V$, there are at most $\ell$ subschemes among $Y_{1}, \ldots, Y_{q}$ which contain $x$.

Remark 1.4. In the case that $Y_{1}=y_{1}, \ldots, Y_{q}=y_{q}$ are points (as in [McKinnon and Roth 2015]), the condition that $y_{1}, \ldots, y_{q}$ are distinct implies that $Y_{1}, \ldots, Y_{q}$ are in 1-subgeneral position (i.e., with $\ell=1$ ).

We establish the following result.

Main Theorem. Let $k$ be a number field and $M_{k}$ be the set of places on $k$. Let $S \subset M_{k}$ be a finite subset containing all archimedean places. Let $V$ be a projective variety defined over $k$ and $Y_{1}, \ldots, Y_{q}$ be closed subschemes of $V$ defined over $k$ in $\ell$-subgeneral position. For any $v \in S$, choose a local Weil function $\lambda_{Y_{j}, v}$ for each $Y_{j}, 1 \leq j \leq q$. Let $L$ be a big line bundle. Then for any $\epsilon>0$

$$
\sum_{v \in S} \sum_{i=1}^{q} \lambda_{Y_{i}, v}(x) \leq \ell\left(\max _{1 \leq i \leq q}\left\{\beta_{L, Y_{i}}^{-1}\right\}+\epsilon\right) h_{L}(x)
$$

holds for all $x$ outside a proper Zariski-closed subset $Z$ of $V(k)$.

The following corollary of our main theorem recovers the main result of [McKinnon and Roth 2015]. The proof will be given in Section 3.

Corollary 1.5 [McKinnon and Roth 2015, Theorem 6.1]. Let $V$ be a projective variety over $k$. Then for any ample line bundle $L$ and any $x \in V(\bar{k})$ either

- $\alpha_{x}(L) \geq \beta_{L, x}$ or 
- there exists a proper subvariety $Z \subset V$, irreducible over $\bar{k}$, with $x \in Z(\bar{k})$ so that $\alpha_{x, V}(L)=\alpha_{x, Z}(L \mid Z)$, i.e., " $\alpha_{x}(L)$ is computed on a proper subvariety of $V^{\prime \prime}$,

where $\alpha_{x}(L)$ is the approximation constant defined in [McKinnon and Roth 2015, Definition 2.9], and $\beta_{L, x}$ is defined in Definition 1.1 (with $Y$ taken as a point $x$ ).

We will show in Lemma 2.2 that for any line bundle $L, x \in V$

$$
\beta_{L, x} \geq \frac{n}{n+1} \epsilon_{x}(L),
$$

where $n=\operatorname{dim} V$. We note that the Seshadri constant $\epsilon_{x}(L)$ does not decrease when restricting to a subvariety [McKinnon and Roth 2015, Proposition 3.4], so we can use induction to further get, from Corollary 1.5 and (1-2), the following result.

Corollary 1.6 [McKinnon and Roth 2015, Theorem 6.2, alternative statement]. Let $V$ be a projective variety over $k$. Let $L$ be any ample line bundle and choose any $x \in V(\bar{k})$. Then for any $\delta>0$, there are only finitely many solutions $y \in V(k)$ to

$$
d_{v}(x, y)<H_{L}(y)^{-\left((n+1) /\left(n \epsilon_{x}(L)\right)+\delta\right)} .
$$

In the case when $V=\mathbb{P}^{n}$ and $L=\mathcal{O}_{\mathbb{P}^{n}}(1)$, we have $\epsilon_{x}(L)=1$ for all $x \in \mathbb{P}^{n}$ (see [McKinnon and Roth 2015, Lemma 3.3]). Therefore the above result generalizes the theorem of Roth.

We now turn to another extreme case when the subschemes $Y_{1}, \ldots, Y_{q}$ are effective Cartier divisors $D_{1}, \ldots, D_{q}$. Let $D:=D_{1}+\cdots+D_{q}$. Assume that each $D_{j}$ is linearly equivalent to a fixed ample divisor $A$. Then we have the following relation of height functions $h_{D}=q h_{A}+O(1)$. On the other hand, by the Riemann-Roch theorem, with $n=\operatorname{dim} V$,

and

$$
h^{0}(N D)=h^{0}(q N A)=\frac{(q N)^{n} A^{n}}{n !}+o\left(N^{n}\right)
$$

Thus

$$
h^{0}\left(N D-m D_{j}\right)=h^{0}((q N-m) A)=\frac{(q N-m)^{n} A^{n}}{n !}+o\left(N^{n}\right) .
$$

$$
\sum_{m \geq 1} h^{0}\left(N D-m D_{j}\right)=\frac{A^{n}}{n !} \sum_{l=0}^{q N-1} l^{n}+o\left(N^{n+1}\right)=\frac{A^{n}(q N-1)^{n+1}}{(n+1) !}+o\left(N^{n+1}\right) .
$$

Hence

$$
\beta_{D, D_{j}}=\lim _{N \rightarrow \infty} \frac{\frac{A^{n}(q N-1)^{n+1}}{(n+1) !}+o\left(N^{n+1}\right)}{N \frac{(q N)^{n} A^{n}}{n !}+o\left(N^{n+1}\right)}=\frac{q}{n+1} .
$$

Thus the Main Theorem, together with the above computation, implies the following result of Chen, Ru, and Yan [2012] (see also [Corvaja and Zannier 2006]). 
Theorem 1.7. Let $k$ be a number field and $M_{k}$ the set of places on $k$. Let $S \subset M_{k}$ be a finite subset containing all archimedean places. Let $V$ be a projective variety of dimension $n$ defined over $k$. Let $D_{1}, \ldots, D_{q}$ be effective Cartier divisors in $\ell$-subgeneral position on $V$. Assume that each $D_{j}, 1 \leq j \leq q$, is linearly equivalent to a fixed ample divisor A. For any $v \in S$, choose a Weil function $\lambda_{D_{j}, v}$ for each $D_{j}, 1 \leq j \leq q$. Then for any $\epsilon>0$

$$
\sum_{v \in S} \sum_{i=1}^{q} \lambda_{D_{i}, v}(x) \leq \ell(n+1+\epsilon) h_{A}(x)
$$

holds for all $x$ outside a proper Zariski-closed subset $Z$ of $V(k)$. In particular, if $D_{1}, \ldots, D_{q}$ are in general position on $V$, then the inequality

$$
\sum_{v \in S} \sum_{i=1}^{q} \lambda_{D_{i}, v}(x) \leq n(n+1+\epsilon) h_{A}(x)
$$

holds for all but finitely many $x \in V(k)$.

In the general case when $D_{1}, \ldots, D_{q}$ are only assumed to be big and nef, we can also compute $\beta_{D, D_{j}}$. The details will be carried out in the next section.

We note that recently the first named author and P. Vojta [2016] obtained the following sharp result in the case when $D_{1}, \ldots, D_{q}$ are in general position and when $V$ is Cohen-Macaulay (for example when $V$ is smooth).

Theorem 1.8 [Ru and Vojta 2016]. Let $k$ be a number field and $M_{k}$ be the set of places on $k$. Let $S \subset M_{k}$ be a finite subset containing all archimedean places. Let $V$ be a projective variety defined over $k$. Assume that $V$ is Cohen-Macaulay. Let $D_{1}, \ldots, D_{q}$ be effective Cartier divisors in general position on $V$. For any $v \in S$, choose a Weil function $\lambda_{D_{j}, v}$ for each $D_{j}, 1 \leq j \leq q$. Let $L$ be a line bundle on $V$ with $h^{0}(V, N L) \geq 1$ for $N$ big enough. Then for any $\epsilon>0$

$$
\sum_{v \in S} \sum_{i=1}^{q} \lambda_{D_{i}, v}(x) \leq\left(\max _{1 \leq i \leq q}\left\{\beta_{L, D_{i}}^{-1}\right\}+\epsilon\right) h_{L}(x)
$$

holds for all $x$ outside a proper Zariski-closed subset $Z$ of $V(k)$.

Theorem 1.8, together with the above computation, recovers the result of [Evertse and Ferretti 2002; 2008] in the case when $V$ is smooth.

\section{Computation of the constant $\beta_{L, Y}$}

We first compute the constant $\beta_{L, y}$, i.e., we let $Y=y$ be a point in $V(k)$. The following lemma is a reformulation of Lemma 4.1 in [McKinnon and Roth 2015]. 
Lemma 2.1. Let $V$ be a projective variety and $x$ be a point in $V$. Let $\pi: \widetilde{V} \rightarrow V$ be the blow-up along $x$, and $E$ be the exceptional divisor. Let $L$ be an ample line bundle and $m$ a positive integer. Then

(i) $h^{0}\left(\tilde{V}, N \pi^{*} L-m E\right)=0$ if $m>N \cdot \gamma_{\mathrm{eff}, x}$, where $\gamma_{\mathrm{eff}, x}$ is defined in [McKinnon and Roth 2015], and

(ii) $h^{0}\left(\tilde{V}, N \pi^{*} L-m E\right) \geq h^{0}(V, N L)-m^{n} \operatorname{mult}_{x} V / n !+O\left(N^{n-1}\right)$ for $N \gg 0$.

Proof. Write $h^{0}\left(\widetilde{V}, N \pi^{*} L-m E\right)=h^{0}\left(\widetilde{V}, N \pi^{*} L-N \cdot \gamma E\right)$, where $\gamma=m / N$. The argument in [McKinnon and Roth 2015] shows that $h^{0}\left(\widetilde{V}, N \pi^{*} L-m E\right) \geq$ $h^{0}(V, N L)-m^{n}$ mult $_{x} V / n !+O\left(N^{n-1}\right)$.

The following is a restatement of Corollary 4.2 in [McKinnon and Roth 2015].

Lemma 2.2. For any ample line bundle $L, x \in V$ and positive integer $m$, we have

$$
\beta_{L, x} \geq \frac{n}{n+1}\left(\frac{L^{n}}{\operatorname{mult}_{x} V}\right)^{1 / n} \geq \frac{n}{n+1} \epsilon_{x}(L) .
$$

Proof. Choose a sufficiently large N. By Lemma 2.1 and the Riemann-Roch theorem,

$$
h^{0}\left(\widetilde{V}, \pi^{*} N L-m E\right) \geq h^{0}(V, N L)\left(1-\frac{\operatorname{mult}_{x} V}{L^{n}}\left(\frac{m}{N}\right)^{n}\right)+O\left(N^{n-1}\right) .
$$

The right-hand side of (2-1) is less than zero when $m>u=\left[N\left(L^{n} / \text { mult }_{x} V\right)^{1 / n}\right]$, hence

$$
\sum_{m=1}^{\infty} h^{0}\left(\tilde{V}, \pi^{*} N L-m E\right) \geq h^{0}(V, N L) \sum_{m=1}^{u}\left(1-\frac{\operatorname{mult}_{x} V}{L^{n}}\left(\frac{m}{N}\right)^{n}\right)+O\left(N^{n}\right) .
$$

Consequently,

$$
\begin{aligned}
\beta_{L, x} & \geq \frac{1}{N} \sum_{m=1}^{u}\left(1-\frac{\text { mult }_{x} V}{L^{n}}\left(\frac{m}{N}\right)^{n}\right)+O\left(\frac{1}{N}\right) \\
& =\frac{1}{N}\left(u-\frac{\text { mult }_{x} V}{L^{n}} \cdot \frac{u^{n+1}}{(n+1) N^{n}}\right)+O\left(\frac{1}{N}\right) \\
& \geq \frac{n u}{(n+1) N}+O\left(\frac{1}{N}\right) .
\end{aligned}
$$

Let $N$ run through all sufficiently large integers. Then we have

$$
\beta_{L, x} \geq \frac{n}{n+1}\left(\frac{L^{n}}{\operatorname{mult}_{x} V}\right)^{1 / n} .
$$

Next we consider the case when $Y_{j}=D_{j}, 1 \leq j \leq q$, are effective big and nef Cartier divisors on $V$. 
Definition 2.3. Suppose $X$ is a complete variety of dimension $n$. Let $D_{1}, \ldots, D_{q}$ be effective Cartier divisors on $X$ and let $D=D_{1}+D_{2}+\cdots+D_{q}$. We say that $D$ has equidegree with respect to $D_{1}, D_{2}, \ldots, D_{q}$ if $D_{i} \cdot D^{n-1}=D^{n} / q$ for all $i=1, \ldots, q$.

Lemma 2.4 [Levin 2009, Lemma 9.7]. Let $V$ be a projective variety of dimension $n$. If $D_{j}, 1 \leq j \leq q$, are big and nef Cartier divisors on $V$, then there exist positive real numbers $r_{j}$ such that $D=\sum_{j=1}^{q} r_{j} D_{j}$ has equidegree.

Since divisors $r_{j} D_{j}$ and $D_{j}$ have the same support, the above lemma tells us that we can always make the given big and nef divisors have equidegree without changing their supports. So now we assume that $D:=D_{1}+\cdots+D_{q}$ is of equidegree. To compute $\beta_{D, D_{j}}$ for $j=1, \ldots, q$, we use the following lemma.

Lemma 2.5 [Autissier 2009, Lemma 4.2]. Suppose E is a big and base-point free Cartier divisor on a projective variety $V$ and $F$ is a nef Cartier divisor on $V$ such that $F-E$ is also nef. Let $\delta>0$ be a positive real number. Then, for any positive integers $N$ and $m$ with $1 \leq m \leq \delta N$, we have

$h^{0}(N F-m E)$

$\geq \frac{F^{n}}{n !} N^{n}-\frac{F^{n-1} \cdot E}{(n-1) !} N^{n-1} m+\frac{(n-1) F^{n-2} \cdot E^{2}}{n !} N^{n-2} \min \left\{m^{2}, N^{2}\right\}+O\left(N^{n-1}\right)$,

where the implicit constant depends on $\beta$.

We compute $\sum_{m>1} h^{0}\left(N D-m D_{i}\right)$ for each $1 \leq i \leq q$. Let $n=\operatorname{dim} V$ and assume that $n \geq 2$. Let $b=D^{n} /\left(n D^{n-1} \cdot D_{i}\right)$ and $A=(n-1) D^{n-2} \cdot D_{i}^{2}$. Then, by Lemma 2.5,

$$
\begin{aligned}
\sum_{m=1}^{\infty} h^{0}(N & \left.D-m D_{i}\right) \\
\geq & \sum_{m=1}^{[b N]}\left(\frac{D^{n}}{n !} N^{n}-\frac{D^{n-1} \cdot D_{i}}{(n-1) !} N^{n-1} m+\frac{A}{n !} N^{n-2} \min \left\{m^{2}, N^{2}\right\}\right)+O\left(N^{n}\right) \\
\geq & \left(\frac{D^{n}}{n !} b-\frac{D^{n-1} \cdot D_{i}}{(n-1) !} \frac{b^{2}}{2}+\frac{A}{n !} g(b)\right) N^{n+1}+O\left(N^{n}\right) \\
& =\left(\frac{b}{2}+\frac{A}{D^{n}} g(b)\right) D^{n} \frac{N^{n+1}}{n !}+O\left(N^{n}\right) \\
& =\left(\frac{b}{2}+\alpha\right) N h^{0}(N D)+O\left(N^{n}\right),
\end{aligned}
$$

where $\alpha:=g(b) A / D^{n}$ and $g: \mathbb{R}^{+} \rightarrow \mathbb{R}^{+}$is the function given by $g(x)=x^{3} / 3$ if $x \leq 1$ and $g(x)=x-\frac{2}{3}$ for $x \geq 1$. Now from the assumption of equidegree $D_{i} \cdot D^{n-1}=D^{n} / q$, so $b=q / n$. Moreover, $\alpha>0$ since $\operatorname{dim} V \geq 2$ and the $D_{i}$ are 
big and nef divisors. Hence

$$
\beta_{D, D_{i}}=\liminf _{N} \frac{\sum_{m \geq 1} h^{0}\left(N D-m D_{i}\right)}{N h^{0}(N D)} \geq \frac{b}{2}+\alpha .
$$

Thus we have proved the following.

Proposition 2.6. Let $V$ be a projective variety of $\operatorname{dim} V \geq 2$ and assume that $D:=\sum_{j=1}^{q} D_{j}$ has equidegree with respect to $D_{1}, \ldots, D_{q}$ which are big and nef. Then

$$
\beta_{D, D_{i}}=\liminf _{N} \frac{\sum_{m \geq 1} h^{0}\left(N D-m D_{i}\right)}{N h^{0}(N D)}>\frac{q}{2 n}+\alpha,
$$

where $\alpha$ is a computable positive number.

Proposition 2.6, together with the Main Theorem, implies the following result.

Theorem 2.7 [Hussein and Ru 2018]. Let $k$ be a number field and let $S \subseteq M_{k}$ be a finite set containing all archimedean places. Let $V$ be a projective variety of dimension $\geq 2$ over $k$ and let $D_{1}, \ldots, D_{q}$ be effective, big, and nef Cartier divisors on $V$ defined over $k$, located in $\ell$-subgeneral position. Let $r_{i}>0$ be real numbers such that $D:=\sum_{i=1}^{q} r_{i} D_{i}$ has equidegree (such numbers exist due to Lemma 2.4). Then, for $\epsilon_{0}>0$ small enough, the inequality

$$
\sum_{v \in S} \sum_{j=1}^{q} r_{j} \lambda_{D_{i}, v}(x)<\ell\left(\frac{2 \operatorname{dim} V}{q}-\epsilon_{0}\right)\left(\sum_{j=1}^{q} r_{j} h_{D_{j}}(x)\right)
$$

holds for all $x \in V(k)$ outside a proper Zariski-closed subset of $V$.

\section{Proof of the Main Theorem}

We first recall some basic properties of local Weil functions associated to closed subschemes from [Silverman 1987, Section 2]. We assume that the readers are familiar with the notion of Weil functions associated to divisors (see [Lang 1983, Chapter 10], [Hindry and Silverman 2000, B.8] or [Silverman 1987, Section 1]).

Let $Y$ be a closed subscheme on a projective variety $V$ defined over $k$. Then one can associate to each place $v \in M_{k}$ a function

$$
\lambda_{Y, v}: V \backslash \operatorname{supp}(Y) \rightarrow \mathbb{R}
$$

satisfying some functorial properties (up to an $M_{k}$-constant) described in [Silverman 1987, Theorem 2.1]. Intuitively, for each $P \in V$ and $v \in M_{k}$,

$$
\lambda_{Y, v}(P)=-\log (v \text {-adic distance from } P \text { to } Y) .
$$

The following lemma indicates the existence of local Weil functions. 
Lemma 3.1. Let $Y$ be a closed subscheme of $V$. There exist effective divisors $D_{1}, \ldots, D_{r}$ such that

$$
Y=\cap D_{i} .
$$

Proof. See Lemma 2.2 from [Silverman 1987].

Definition 3.2. Let $k$ be a number field, and $M_{k}$ be the set of places on $k$. Let $V$ be a projective variety over $k$ and let $Y \subset V$ be a closed subscheme of $V$. We define the (local) Weil function for $Y$ with respect to $v \in M_{k}$ as

$$
\lambda_{Y, v}=\min _{i}\left\{\lambda_{D_{i}, v}\right\},
$$

when $Y=\cap D_{i}$ (such $D_{i}$ exist according to the above lemma).

Lemma 3.3 [Vojta 1987, Lemma 2.5.2; Silverman 1987, Theorem 2.1(h)]. Let $Y$ be a closed subscheme of $V$ and let $\widetilde{V}$ be a blow-up of $V$ along $Y$ with exceptional divisor $E=\pi^{*} Y$. Then $\lambda_{Y, v}(\pi(P))=\lambda_{E, v}(P)+O_{v}(1)$ for $P \in \widetilde{V}$.

Note that in the original statement of Lemma 2.5.2 in [Vojta 1987], $V$ is assumed to be smooth, but from the proof it is easy to see that it works for a general projective variety from Theorem 2.1(h) in [Silverman 1987].

For our purpose, it suffices to fix a choice of local Weil functions $\lambda_{Y_{i}, v}$ for each $1 \leq i \leq q$ and $v \in S$.

Lemma 3.4. Let $Y_{1}, \ldots, Y_{q}$ be closed subschemes of a projective variety $V$ in $\ell$-subgeneral position. Then

$$
\sum_{i=1}^{q} \lambda_{Y_{i}, v}(x) \leq \max _{I} \sum_{j \in I} \lambda_{Y_{j}, v}(x)+O_{v}(1),
$$

where I runs over all index subsets of $\{1, \ldots, q\}$ with $\ell$ elements for all $x \in V(k)$. Proof. Let $\left\{i_{1}, \ldots, i_{q}\right\}=\{1, \ldots, q\}$. Since the $Y_{i}, 1 \leq i \leq q$, are in $\ell$-subgeneral position, $\bigcap_{t=1}^{\ell+1} Y_{i_{t}}=\varnothing$. Then

$$
\min _{1 \leq i \leq \ell+1}\left\{\lambda_{Y_{i}, v}\right\}=\left\{\lambda \cap_{t=1}^{\ell+1} Y_{i_{t}, v}\right\}=O_{v}(1) .
$$

We note that the first equality follows from (3-1), the definition of the local Weil function; and the second equality follows from Corollary 3.3 in [Lang 1983, Chapter 10]. For $x$ with the following ordering

we have

$$
\lambda_{Y_{i_{1}}, v}(x) \geq \lambda_{Y_{i_{2}}, v}(x) \geq \cdots \geq \lambda_{Y_{i_{q}}, v}(x),
$$

$$
\sum_{i=1}^{q} \lambda_{Y_{i}, v}(x)=\sum_{i=1}^{\ell} \lambda_{Y_{i}, v}(x)+O_{v}(1) .
$$

Then assertion (3-2) follows directly as the number of subvarieties under consideration is finite. 
We also need the following generalized Schmidt subspace theorem.

Theorem 3.5 [Ru and Vojta 2016, Theorem 2.7]. Let $k$ be a number field, $S$ be a finite set of places of $k$ containing all archimedean places, $X$ be a complete variety over $k, D$ be a Cartier divisor on $X, W$ be a nonzero linear subspace of $H^{0}(X, \mathcal{O}(D)), s_{1}, \ldots, s_{q}$ be nonzero elements of $W, \epsilon>0$, and $c \in \mathbb{R}$. For each $j=1, \ldots, q$, let $D_{j}$ be the Cartier divisor $\left(s_{j}\right)$ and $\lambda_{D_{j}}$ be a Weil function for $D_{j}$. Then there is a proper Zariski-closed subset $Z$ of $X$, depending only on $k, S, X$, $D, W, s_{1}, \ldots, s_{q}, \epsilon, c$, and the choices of Weil and height functions, such that the inequality

$$
\sum_{v \in S} \max _{J} \sum_{j \in J} \lambda_{D_{j}, v}(x) \leq(\operatorname{dim} W+\epsilon) h_{D}(x)+c
$$

holds for all $x \in(X \backslash Z)(k)$. Here the set $J$ ranges over all subsets of $\{1, \ldots, q\}$ such that the sections $\left(s_{j}\right)_{j \in J}$ are linearly independent.

We are now ready to prove the Main Theorem.

Proof of the Main Theorem. Let $\delta>0$ be a sufficiently small number. We may choose a sufficiently large integer $N$ such that, for $i=1, \ldots, q$,

$$
\sum_{m=1}^{\infty} h^{0}\left(\widetilde{V}_{i}, N \pi^{*} L-E_{i}\right) \geq\left(\beta_{L, Y_{i}}-\delta\right) N h^{0}(V, N L),
$$

where $\pi_{i}: \widetilde{V}_{i} \rightarrow V$ is the blow-up at $Y_{i}$ and $E_{i}=\pi^{-1}\left(Y_{i}\right)$ is he exceptional divisor of $\pi_{i}$.

Let $x \in V(k)$ and $v \in S$. Since the $Y_{i}, 1 \leq i \leq q$, are in $\ell$-subgeneral position, it follows from Lemma 3.4 that

$$
\sum_{i=1}^{q} \lambda_{Y_{i}, v}(x) \leq \ell \lambda_{Y_{i_{0}}, v}(x)+O_{v}(1),
$$

for some $i_{0}$ with $1 \leq i_{0} \leq q$, where the constant $O_{v}(1)$ is independent of $x$. Note that $i_{0}$ depends on the point $x$, but $O_{v}(1)$ is independent of $x$.

Write $\widetilde{V}_{i_{0}}$ as $\widetilde{V}, \pi_{i_{0}}$ as $\pi$ and $E_{i_{0}}$ as $E$. We consider the following filtration.

$$
H^{0}\left(\tilde{V}, \pi^{*} N L\right) \supseteq H^{0}\left(\tilde{V}, \pi^{*} N L-E\right) \supseteq H^{0}\left(\tilde{V}, \pi^{*} N L-2 E\right) \supseteq \cdots
$$

We identify $H^{0}(V, N L)$ with $H^{0}\left(\widetilde{V}, \pi^{*} N L\right)$ as vector spaces (note: according to the footnote on page 553 in [McKinnon and Roth 2015], if $X$ is not normal, then $H^{0}(V, N L)$ may only be a proper subspace of $H^{0}\left(\tilde{V}, \pi^{*} N L\right)$. However, since the volume is a birational constant, the asymptotic calculations go through without change). Choose regular sections $s_{1}, \ldots, s_{M} \in H^{0}(V, N L)$ successively so that their pull-back $\pi^{*} s_{1}, \ldots, \pi^{*} s_{M} \in H^{0}\left(\tilde{V}, \pi^{*} N L\right)$ form a basis associated to this 
filtration, where $M=h^{0}\left(\widetilde{V}, N \pi^{*} L\right)$. For a section $\pi^{*} s \in H^{0}\left(\widetilde{V}, \pi^{*} N L-m E\right)$ (regarded as a subspace of $H^{0}\left(\widetilde{V}, \pi^{*} N L\right)$ ) we have

$$
\operatorname{div}\left(\pi^{*} s\right) \geq m E \text {. }
$$

Hence, $\lambda_{\left(\pi^{*} s\right), v} \geq m \lambda_{E, v}+O_{v}(1)$. Note that although $O_{v}(1)$ here depends on $i_{0}$ (which depends on $x$ ), there are $q$ choices of such $i_{0}$ and $V$ is compact, so we can again make $O_{v}(1)$ independent of $x$. Therefore, also using Lemma 3.3 and (3-5),

$$
\begin{aligned}
\sum_{j=1}^{M} \lambda_{\left(\pi^{*} s_{j}\right), v} & \\
& \geq \sum_{m=1}^{\infty} m\left(h^{0}\left(\tilde{V}, \pi^{*} N L-m E\right)-h^{0}\left(\tilde{V}, \pi^{*} N L-(m+1) E\right)\right) \lambda_{E, v}+O_{v}(1) \\
& =\sum_{m=1}^{\infty} m\left(h^{0}\left(\tilde{V}, \pi^{*} N L-m E\right)-h^{0}\left(\tilde{V}, \pi^{*} N L-(m+1) E\right)\right) \lambda_{Y_{i_{0}}, v} \circ \pi+O_{v}(1) \\
& =\sum_{m=1}^{\infty} h^{0}\left(\tilde{V}, \pi^{*} N L-m E\right) \lambda_{Y_{i_{0}}, v} \circ \pi+O_{v}(1) \\
& \geq\left(\beta_{L, Y_{i_{0}}}-\delta\right) N h^{0}(V, N L) \lambda_{Y_{i_{0}, v}} \circ \pi+O_{v}(1) .
\end{aligned}
$$

The functorial property of Weil functions implies $\lambda_{\left(\pi^{*} s_{j}\right), v}=\lambda_{\left(s_{j}\right), v} \circ \pi+O_{v}(1)$. Hence, the above inequality, together with (3-6), implies that

$$
\begin{aligned}
& \sum_{i=1}^{q} \lambda_{Y_{i}, v}(x) \\
& \quad \leq \frac{\ell}{N \cdot h^{0}(V, N L)\left(\min _{1 \leq i \leq q}\left\{\beta_{L, Y_{i}}\right\}-\delta\right)} \max _{J}\left\{\sum_{j \in J} \lambda_{\left(s_{j}\right), v}(x)\right\}+O_{v}(1),
\end{aligned}
$$

where $J$ is a subset containing $M$ linearly independent sections taken among the collection of sections $\left\{s_{j}\left(i_{0}, v\right) \mid 1 \leq i_{0} \leq q, v \in S\right\}$ coming from the claim (3-6). It then follows from Theorem 3.5 and a suitable choice of $\delta$ that for a given $\epsilon>0$ there exists a proper algebraic subset $Z$ of $V$ defined over $k$ such that

$$
\sum_{v \in S} \sum_{i=1}^{q} \lambda_{Y_{i}, v}(x) \leq\left(\ell \cdot \max _{1 \leq i \leq q}\left\{\beta_{L, Y_{i}}^{-1}\right\}+\epsilon\right) h_{L}(x),
$$

for all $x \in V(k) \backslash Z(k)$.

Proof of Corollary 1.5. Let $v$ be a place of $k$. The main point of the proof is to reformulate the distance function $d_{v}(\cdot, \cdot)$ defined on $V(\bar{k})$ [McKinnon and Roth 2015 , Section 2] into a product of several distance functions on $V(K)$, where $K$ is a finite extension of $k$. Following the construction in [McKinnon and Roth 2015, 
Section 2], we fix an extension of $v$ to $\bar{k}$. The place defines an absolute value $\|\cdot\|_{v}$ on $\bar{k}$. If $K \subset \bar{k}$ is a finite extension of $k$, then $d_{v}(\cdot, \cdot)_{K}=d_{v}(\cdot, \cdot)_{k}^{\left[K_{v}: k_{v}\right]}$. Here $d_{v}(\cdot, \cdot)_{K}$ refers to the distance function defined by using the same embedding and normalizing with respect to $K$ and $d_{v}(\cdot, \cdot)_{k}$ the distance function normalized with respect to $k$ (see [McKinnon and Roth 2015, Proposition 2.1(b)]). Assume that $V \subset \mathbb{P}^{N}$ (given by the canonical map associated to the ample line bundle $L$ ). For a given fixed point $y=\left[y_{0}: \cdots: y_{N}\right] \in V(\bar{k})$, let $K$ be the Galois closure of $k\left(y_{0}, \ldots, y_{N}\right)$ over $k$. For each $v \in M_{k}$, the inclusion map $\left.\left(i_{v}\right)\right|_{K}: K \rightarrow \bar{k}_{v}$ induces a place $w_{0}:=v$ of $K$ over $v$, and other places $w$ of $K$ over $v$ are conjugates by elements $\sigma_{w} \in \operatorname{Gal}(K / k)$ such that $\left\|\sigma_{w}(a)\right\|_{w}=\|a\|_{v}$ for all $a \in K$. Then, for $x, y \in K$,

$$
\begin{aligned}
\prod_{w \in M_{K}, w \mid v} d_{w}\left(\sigma_{w}(x), \sigma_{w}(y)\right)_{K} & =\prod_{w \in M_{K}, w \mid v} d_{v}(x, y)_{K} \\
& =\prod_{w \in M_{K}, w \mid v} d_{v}(x, y)_{k}^{\left[K_{v}: k_{v}\right]} \\
& =[K: k] d_{v}(x, y)_{k},
\end{aligned}
$$

i.e.,

$$
d_{v}(x, y)_{k}=\prod_{w \in M_{K}, w \mid v} d_{w}\left(\sigma_{w}(x), \sigma_{w}(y)\right)_{K}^{1 /[K: k]}, \quad \text { for } x, y \in K .
$$

To compute $\alpha_{y}(L)$, we consider any sequence $\left\{x_{i}\right\} \subseteq V(k)$ of distinct points with $d_{v}\left(y, x_{i}\right) \rightarrow 0$. By (3-11), we have $d_{v}\left(y, x_{i}\right)_{k}=\prod_{w \in M_{K}, w \mid v} d_{w}\left(\sigma_{w}(y), x_{i}\right)_{K}^{1 /[K: k]}$. (Here we extend $\sigma_{w} \in \operatorname{Gal}(K / k)$ to the map from $V(K)$ to $V(K)$ by acting on the coordinates of the points.) The distance function $d_{w}(y, x)$ in [McKinnon and Roth $2015]$ is constructed by choosing an embedding $\phi_{L}: V \rightarrow \mathbb{P}^{N}$ into a projective space via the sections of $L$ and measuring the distance in the embedded space. For a fixed $y$ we denote $-\log d_{w}(y, \cdot)$ by $\lambda_{\phi(y), w}$, which is a local Weil function on the embedded space. We note that this fact can also be proved by a slight modification of Lemma 2.6 in [McKinnon and Roth 2015]. By the functoriality of Weil functions of closed subschemes [Silverman 1987, Theorem 2.1(h)] we have $-\log d_{w}\left(\sigma_{w}(y), x\right)=$ $\lambda_{\sigma_{w}(y), w}(x)+O(1)$. On the other hand, it is clear from the definition that $\beta_{L, y}=$ $\beta_{L, \sigma_{w}(y)}$ for very $\sigma_{w} \in \operatorname{Gal}(K / k)$. The Main Theorem then implies that for any $\epsilon>0$

$$
\log d_{v}\left(y, x_{i}\right)=\frac{1}{[K: k]} \sum_{w \in M_{K}, w \mid v} \log d_{w}\left(y, x_{i}\right) \geq-\left(\left\{\beta_{L, y}^{-1}\right\}+\epsilon\right) h_{L}\left(x_{i}\right)
$$

holds for all $x_{i}$ outside a proper Zariski-closed subset $Z$ of $V(K)$ (note that, in this case, $\ell=1$ ). We note that $Z$ is indeed defined over $k$ since all the $x_{i}$ are in $k$. In conclusion, we have shown that for all sequences $\left\{x_{i}\right\} \subseteq V(k)$ of distinct points with $d_{v}\left(y, x_{i}\right) \rightarrow 0$, if $\alpha_{y}\left(\left\{x_{i}\right\}, L\right)<\beta_{L, y}$, then all but finitely many of the points of $\left\{x_{i}\right\}$ lie in $Z$. If (a) holds, then we are done. Therefore we assume that $\alpha_{y}(L)>\beta_{L, y}$. 
Then the previous conclusion shows, in this case, that $\alpha_{y}(L)=\alpha_{y, Z}\left(\left.L\right|_{Z}\right)$. To see $Z$ is irreducible over $\bar{k}$, we first use Proposition 2.14(f) in [McKinnon and Roth 2015] to reduce $Z$ to one of the irreducible components of $Z$ over $k$, say $Y$ such that $\alpha_{y, Z}\left(\left.L\right|_{Z}\right)=\alpha_{y, Y}\left(\left.L\right|_{Y}\right)$. Without loss of generality we can assume that $Z=Y$, i.e., $Z$ itself is irreducible over $k$. We then apply Lemma 2.17 in [McKinnon and Roth 2015] to conclude that $Z$ is indeed geometrically irreducible, i.e., $Z$ is irreducible over $\bar{k}$.

\section{The complex case}

In this section, we consider the analogous result of our Main Theorem in Nevanlinna theory. Let $V$ be a complex projective variety. We use the standard notation in Nevanlinna theory (see, for example, [Ru 2016]). Note that the Weil function for divisors has been defined, so the Weil function $\lambda_{Y}$ for a subscheme $Y \subset V$ can also be defined using Lemma 3.1, similar to Definition 3.2. We define, for a holomorphic map $f: \mathbb{C} \rightarrow V$ with $f(\mathbb{C}) \not \subset Y$, the proximity function

$$
m_{f}(r, Y)=\int_{0}^{2 \pi} \lambda_{Y}\left(f\left(r e^{i \theta}\right)\right) \frac{d \theta}{2 \pi} .
$$

We note that all the properties used above about the Weil functions in the arithmetic case hold for the complex case (see, for example, [Ru 2016; Ru and Vojta 2016]).

Theorem 4.1. Let $V$ be a complex projective variety and $Y_{1}, \ldots, Y_{q}$ be closed subschemes of $V$ in $\ell$-subgeneral position. Let L be a big line bundle. Let $f: \mathbb{C} \rightarrow V$ be a holomorphic map with Zariski dense image. Then for any $\epsilon>0$

$$
\sum_{i=1}^{q} m_{f}\left(r, Y_{i}\right) \leq \ell\left(\max _{1 \leq i \leq q}\left\{\beta_{L, Y_{i}}^{-1}\right\}+\epsilon\right) T_{f, L}(r) \|,
$$

where $\|$ means that the inequality holds for all $r \in(0,+\infty)$ outside a set of finite Lebesgue measure.

To prove the theorem, we need the following result.

Theorem 4.2 [Ru and Vojta 2016, Theorem 2.8]. Let X be a complex projective variety, $D$ be a Cartier divisor on $X, W$ be a nonzero linear subspace of $H^{0}(X, \mathcal{O}(D))$, and $s_{1}, \ldots, s_{q}$ be nonzero elements of $W$. Let $f: \mathbb{C} \rightarrow X$ be a holomorphic map with Zariski-dense image. Then

$\int_{0}^{2 \pi} \max _{J} \sum_{j \in J} \lambda_{\left(s_{j}\right)}\left(f\left(r e^{i \theta}\right)\right) \frac{d \theta}{2 \pi} \leq(\operatorname{dim} W) T_{f, D}(r)+O\left(\log ^{+} T_{f, D}(r)\right)+o(\log r) \|$,

where the set $J$ ranges over all subsets of $\{1, \ldots, q\}$ such that the sections $\left(s_{j}\right)_{j \in J}$ are linearly independent. 
Proof of Theorem 4.1. Similar to the proof of the Main Theorem, let $\delta>0$ be a sufficiently small number. We choose $N$ large enough that, for $i=1, \ldots, q$,

$$
\sum_{m=1}^{\infty} h^{0}\left(\widetilde{V}_{i}, N \pi_{i}^{*} L-m E_{i}\right) \geq\left(\beta_{L, Y_{i}}-\delta\right) N h^{0}(V, N L) .
$$

Let $x \in V$. Since $Y_{i}, 1 \leq i \leq q$, are in $\ell$-subgeneral position, similar to Lemma 3.4, we have

$$
\sum_{i=1}^{q} \lambda_{Y_{i}}(x) \leq \ell \lambda_{Y_{i_{0}}}(x)+O(1)
$$

for some $i_{0}$ with $1 \leq i_{0} \leq q$, where $i_{0}$ depends on the point $x$, but $O(1)$ is independent of $x$.

Let $\pi: \widetilde{V} \rightarrow V$ be the blow-up at $Y_{i_{0}}$ and $E=\pi^{-1}\left(Y_{i_{0}}\right)$ be the exceptional divisor of $\pi$. We consider the filtration of $H^{0}\left(\widetilde{V}, \pi^{*} N L\right)$ defined in (3-7). By identifying $H^{0}(V, N L)$ with $H^{0}\left(\widetilde{V}, \pi^{*} N L\right)$ as vector spaces, we can choose regular sections $s_{1}, \ldots, s_{M} \in H^{0}(V, N L)$, where $M=h^{0}(V, N L)$, successively so that their pullbacks $\pi^{*} s_{1}, \ldots, \pi^{*} s_{M} \in H^{0}\left(\widetilde{V}, \pi^{*} N L\right)$ form a basis associated to this filtration. Then, in the same way as deriving (3-9), we can get

$$
\sum_{i=1}^{q} \lambda_{Y_{i}}(x) \leq \frac{\ell}{N \cdot h^{0}(V, N L)\left(\min _{1 \leq i \leq q}\left\{\beta_{L, Y_{i}}\right\}-\delta\right)} \sum_{j=1}^{q} \lambda_{\left(s_{j}\right)}(x)+O(1) .
$$

Note that the basis $\left\{s_{1}, \ldots, s_{M}\right\}$ depends only on $i_{0}$, so the number of such choices is finite, since $i_{0} \in\{1, \ldots, q\}$, while $x$ varies in (4-2). We denote the set of bases as $J_{1}, \ldots, J_{T}$. Thus we get, for every $x \in V$,

$$
\sum_{i=1}^{q} \lambda_{Y_{i}}(x) \leq \frac{\ell}{N \cdot h^{0}(V, N L)\left(\min _{1 \leq i \leq q}\left\{\beta_{L, Y_{i}}\right\}-\delta\right)} \max _{1 \leq t \leq T} \sum_{j \in J_{t}} \lambda_{\left(s_{j}\right)}(x)+O(1) .
$$

By taking $x=f\left(r e^{i \theta}\right)$ and then integrating, it then follows from Theorem 4.2 and a suitable choice of $\delta$ that, for the given $\epsilon>0$,

$$
\sum_{i=1}^{q} \int_{0}^{2 \pi} \lambda_{Y_{i}}\left(f\left(r e^{i \theta}\right)\right) \frac{d \theta}{2 \pi} \leq \ell\left(\max _{1 \leq i \leq q}\left\{\beta_{\mathcal{L}, Y_{i}}^{-1}\right\}+\epsilon\right) T_{f, \mathcal{L}}(r) \| .
$$

This finishes the proof.

Theorem 4.1, together with Lemma 2.2, implies the following corollary.

Corollary 4.3. Let $V$ be a complex projective variety of dimension $n$ and $a_{1}, \ldots, a_{q}$ be distinct points on $V$. Let $L$ be an ample line bundle. Let $f: \mathbb{C} \rightarrow V$ be a 
holomorphic map with Zariski dense image. Then for any $\epsilon>0$,

$$
\sum_{i=1}^{q} m_{f}\left(r, a_{i}\right) \leq\left(\frac{n+1}{n} \max _{1 \leq i \leq q}\left\{\epsilon_{a_{i}}^{-1}(L)\right\}+\epsilon\right) T_{f, L}(r) \|,
$$

where $\epsilon_{x}(L)$ is the Seshadri constant of $L$ at the point $x \in V$.

In particular, if $V=\mathbb{P}^{n}$, then for any $\epsilon>0$,

$$
\sum_{i=1}^{q} m_{f}\left(r, a_{i}\right) \leq\left(\frac{n+1}{n}+\epsilon\right) T_{f, L}(r) \| .
$$

\section{Acknowledgments}

The first named author wishes to thank the Institute of Mathematics, Academia Sinica, Taiwan for kind hospitality during which part of the work on this paper took place. The authors thank Shin-Yao Jow for pointing out a mistake in an earlier version and helpful discussions. The authors also wish to thank the referee for many helpful suggestions.

\section{References}

[Autissier 2009] P. Autissier, "Géométries, points entiers et courbes entières", Ann. Sci. Éc. Norm. Supér. (4) 42:2 (2009), 221-239. MR Zbl

[Chen et al. 2012] Z. Chen, M. Ru, and Q. Yan, "The degenerated second main theorem and Schmidt's subspace theorem", Sci. China Math. 55:7 (2012), 1367-1380. MR Zbl

[Corvaja and Zannier 2004] P. Corvaja and U. Zannier, “On a general Thue's equation”, Amer. J. Math. 126:5 (2004), 1033-1055. MR Zbl

[Corvaja and Zannier 2006] P. Corvaja and U. Zannier, "Addendum to 'On a general Thue's equation'”, Amer. J. Math. 128:4 (2006), 1057-1066. MR Zbl

[Evertse and Ferretti 2002] J.-H. Evertse and R. G. Ferretti, "Diophantine inequalities on projective varieties", Int. Math. Res. Not. 2002:25 (2002), 1295-1330. MR Zbl

[Evertse and Ferretti 2008] J.-H. Evertse and R. G. Ferretti, "A generalization of the Subspace Theorem with polynomials of higher degree", pp. 175-198 in Diophantine approximation, edited by H. P. Schlickewei et al., Dev. Math. 16, Springer, 2008. MR Zbl

[Hindry and Silverman 2000] M. Hindry and J. H. Silverman, Diophantine geometry, Graduate Texts in Mathematics 201, Springer, 2000. MR Zbl

[Hussein and $\mathrm{Ru} 2018$ ] S. Hussein and M. Ru, "A general defect relation and height inequality for divisors in subgeneral position", preprint, 2018. To appear in Asian Journal of Mathematics.

[Lang 1983] S. Lang, Fundamentals of Diophantine geometry, Springer, 1983. MR Zbl

[Levin 2009] A. Levin, "Generalizations of Siegel's and Picard's theorems”, Ann. of Math. (2) 170:2 (2009), 609-655. MR Zbl

[Levin 2014] A. Levin, "On the Schmidt subspace theorem for algebraic points", Duke Math. J. 163:15 (2014), 2841-2885. MR Zbl

[McKinnon and Roth 2015] D. McKinnon and M. Roth, "Seshadri constants, diophantine approximation, and Roth's theorem for arbitrary varieties", Invent. Math. 200:2 (2015), 513-583. MR Zbl 
[Ru 2016] M. Ru, "A defect relation for holomorphic curves intersecting general divisors in projective varieties”, J. Geom. Anal. 26:4 (2016), 2751-2776. MR Zbl

[Ru 2017] M. Ru, “A general Diophantine inequality”, Funct. Approx. Comment. Math. 56:2 (2017), 143-163.

[Ru and Vojta 2016] M. Ru and P. Vojta, "A birational Nevanlinna constant and its consequences", preprint, 2016. arXiv

[Silverman 1987] J. H. Silverman, "Arithmetic distance functions and height functions in Diophantine geometry”, Math. Ann. 279:2 (1987), 193-216. MR Zbl

[Vojta 1987] P. Vojta, Diophantine approximations and value distribution theory, Lecture Notes in Mathematics 1239, Springer, 1987. MR Zbl

Communicated by Joseph $\mathrm{H}$. Silverman

Received 2016-09-01 Revised 2017-02-06 Accepted 2017-03-31

minru@math.uh.edu Department of Mathematics,

East China Normal University, Shanghai, China

Department of Mathematics, University of Houston, Houston, TX, United States

jwang@math.sinica.edu.tw Institute of Mathematics, Academia Sinica, Taipei, Taiwan 


\section{Algebra \& Number Theory}

msp.org/ant

\section{EDITORS}

MANAGING EDITOR

Bjorn Poonen

Massachusetts Institute of Technology

Cambridge, USA

\author{
EDITORIAL BOARD CHAIR \\ David Eisenbud \\ University of California \\ Berkeley, USA
}

BOARD OF EDITORS

Richard E. Borcherds

J-L. Colliot-Thélène

Brian D. Conrad

Samit Dasgupta

Hélène Esnault

Gavril Farkas

Hubert Flenner

Sergey Fomin

Edward Frenkel

Andrew Granville

Joseph Gubeladze

Roger Heath-Brown

Craig Huneke

Kiran S. Kedlaya

János Kollár

Yuri Manin

Philippe Michel

Susan Montgomery

Shigefumi Mori
University of California, Berkeley, USA

CNRS, Université Paris-Sud, France

Stanford University, USA

University of California, Santa Cruz, USA

Freie Universität Berlin, Germany

Humboldt Universität zu Berlin, Germany

Ruhr-Universität, Germany

University of Michigan, USA

University of California, Berkeley, USA

Université de Montréal, Canada

San Francisco State University, USA

Oxford University, UK

University of Virginia, USA

Univ. of California, San Diego, USA

Princeton University, USA

Northwestern University, USA

École Polytechnique Fédérale de Lausanne

University of Southern California, USA

RIMS, Kyoto University, Japan
Martin Olsson

Raman Parimala

Jonathan Pila

Anand Pillay

Michael Rapoport

Victor Reiner

Peter Sarnak

Joseph H. Silverman

Michael Singer

Christopher Skinner

Vasudevan Srinivas

J. Toby Stafford

Pham Huu Tiep

Ravi Vakil

Michel van den Bergh

Marie-France Vignéras

Kei-Ichi Watanabe

Shou-Wu Zhang
University of California, Berkeley, USA

Emory University, USA

University of Oxford, UK

University of Notre Dame, USA

Universität Bonn, Germany

University of Minnesota, USA

Princeton University, USA

Brown University, USA

North Carolina State University, USA

Princeton University, USA

Tata Inst. of Fund. Research, India

University of Michigan, USA

University of Arizona, USA

Stanford University, USA

Hasselt University, Belgium

Université Paris VII, France

Nihon University, Japan

Princeton University, USA

PRODUCTION

production@msp.org

Silvio Levy, Scientific Editor

See inside back cover or msp.org/ant for submission instructions.

The subscription price for 2017 is US $\$ 325 /$ year for the electronic version, and $\$ 520 /$ year ( $\$ 55$, if shipping outside the US) for print and electronic. Subscriptions, requests for back issues and changes of subscriber address should be sent to MSP.

Algebra \& Number Theory (ISSN 1944-7833 electronic, 1937-0652 printed) at Mathematical Sciences Publishers, 798 Evans Hall \#3840, c/o University of California, Berkeley, CA 94720-3840 is published continuously online. Periodical rate postage paid at Berkeley, CA 94704, and additional mailing offices.

ANT peer review and production are managed by EditFLOW ${ }^{\circledR}$ from MSP.

\section{PUBLISHED BY}

mathematical sciences publishers

nonprofit scientific publishing

http://msp.org/

(C) 2017 Mathematical Sciences Publishers 


\section{Algebra \& Number Theory}

Volume $11 \quad$ No. $10 \quad 2017$

Tate cycles on some unitary Shimura varieties mod $p$

David Helm, Yichao Tian and Liang XiaO

Complex conjugation and Shimura varieties

DON BLASIUS and LUCIO GUERBEROFF

A subspace theorem for subvarieties

Min RU and JULIE TZU-YUEH WANG

Francesc Castella, Chan-Ho Kim and Matteo Longo

MARCo PIZZATO, TARo SANO and LUCA TASIN

Generalized Kuga-Satake theory and good reduction properties of Galois representations

STEFAN PATRIKIS

Remarks on the arithmetic fundamental lemma

CHAO LI and YIHANG ZHU 